\title{
Evaluation of dorsal fat and body condition in sows that use measurements and morph metric indexes
}

\begin{abstract}
The objective of the study was to build a model to estimate body condition by dorsal fat and zoomometric measurements; Thus, 133 Yorkshire sows aged 6-12, 13-18 and older than 18 months were evaluated, evaluating different zoomometric measurements such as: raised to the cross (AC), raised to the rump (AEG), width of the rear rump (IIAS), body length (LEI), sternal back elevation (ADE), thoracic perimeter (PRT), previous reed perimeter (PCA) and bicostal perimeter (PBC); At the same time, the thickness of the dorsal fat located at the level of the last rib was obtained through ultrasound (linear probe at $6.5 \mathrm{Mhz}$ ) at 4.6 and $8 \mathrm{~cm}$ from the midline both to The right side and to the left side. All these data were subjected to a normality test that allowed to know that it was a parametric analysis, followed by a Pearson correlation study and finally to multiple regression analysis that yielded several equation models to obtain the value of dorsal fat and by means of this value estimate body condition; Selecting the model that best fits the parameters studied obtained within it the variable AC, PRT and number of births.
\end{abstract}

Volume 7 Issue 4 - 2018

\author{
Oscar Patricio Núñez Torres \\ Faculty of Agricultural Sciences, Technical University of Ambato, \\ Ecuador
}

\begin{abstract}
Correspondence: Oscar Patricio Núñez Torres, Career in Veterinary Medicine and Zootechnics, Faculty of Agricultural Sciences, Technical University of Ambato, Ambato, Ecuador, Tel (593)032746171,Email op.nunez@uta.edu.ec
\end{abstract}

Received: April 09, 2018 | Published: July 26, 2018

Keywords: Body condition, dorsal fat, zoomometric measurements.

\section{Introduction}

Currently, the national pork production has been changing and improving drastically, with the end of being able to offer the market a product for human consumption of high quality health and nutritional status, low productive systems that generate profitability through efficient work, organized and focused on agricultural best management practices. ${ }^{1}$ In the provinces of Chimborazo and Tungurahua pig production in the country has become important in recent years. The pork is the third largest source of protein of animal origin and according to the third national agricultural census 1 of every 2 productive units possessed some type of hog production, of which $90 \%$ are raised in traditional management conditions. ${ }^{2}$ In the market there are very specialized lines within the different races, covering productive skills, reproductive, and quality of the flesh; which allows you to get the most suitable according to the demand that is present in the market, which means that usually the selection of genotypes pigs takes place in the greater efficiency of deposition of muscle tissue which is based on the measurement of the depth of the subcutaneous adipose tissue dorsal level. ${ }^{3}$ On the other hand the zoometria is charged with studying the measures in the different regions of the body of animals and clarify the relationship between them and their economic value for its exploitation. ${ }^{4}$ As a general rule the young animals have greater development in the extremities while adults show their development in the measures of the trunk; why varies the lift of the cross and their relative proportions, as is the growth of the animal, the race to which it belongs, the power class to which it has been subjected, etc. ${ }^{5}$

The possibility of assessing the thickness of fat in vivo using image analysis would be very useful in the pig sector Yorkshire specifically in females since this technique is more objective and allows to achieve a more real and not just rely on the estimation of the body condition by visual means, however one of the problems with this technique is that it is depending on the technician that applies and even of the ultrasound machine that is used, ${ }^{6}$ another point is that these results sometimes do not coincide with those of the channel due to the fact that the pig in vivo is constantly providing heat what makes to the sonograph vary the consistency of the grease, ${ }^{7}$ Despite this ultrasound provides a very good to excellent prediction of these characters, much more acceptable than the measure itself of the channel. It has also been pointed out that the measurement of the thickness of the backfat is more accurate than the area of the Longissimus dorsi. ${ }^{8}$ Well the extent of the backfat allows us to improve supply levels correct, obtain low mortality of bristles, achieve a weaning-reduced service interval, increasing the total born and living, greater income from slaughtered animals, reduction of non-productive days, improvement in the development of new moms, improvement in the rate of births, lower rates of replacement. ${ }^{9}$ In order to optimize the reproductive behavior of the show is necessary to establish a management strategy and power based on the control of the body condition of each individual, therefore the fat can be increased through the restriction of dietary protein, but the contribution of this during the reproductive management specifically before puberty is also necessary in order to avoid the negative effects that protein restriction has on the age at which it reaches puberty or on the ovulation rate. ${ }^{10}$ To be able to predict the body condition of animals in vivo is of great utility for breeding programs and incidence of environmental factors at the time of the slaughter in pigs. Taking into account that the relationship muscle-bone is variable between animals, the degree of thickening is the main factor that determines the performance of lean meat. To increase the proportion of fat decreases the proportion of muscle. ${ }^{11}$ It is also of great importance as a criterion for the selection of replacement females why this research lies in estimating the fat and the body condition of sows using morphometric measures and indices. 


\section{Materials and methods}

The research along with the data collection took place in two provinces, in Chimborazo within which evaluated different farms located in the Canton Alausí parish Huigra presented the same temperatures ranging from $16^{\circ} \mathrm{C}$ to $26^{\circ} \mathrm{C}$ and an altitude of 1331 meters above sea level, in the canton of Zunag Chunchi parish with temperatures ranging between $14^{\circ} \mathrm{C}$ and $21^{\circ} \mathrm{C}$ and an altitude of 2177 meters above sea level. In the province of Tungurahua in the canton Cevallos Querochaca sector, Technical University of Ambato and hog farm sector Andignato PROIMBA with temperature of $18^{\circ} \mathrm{C}$ and an altitude of 2865 meters above sea level, in the canton of Pillaro Huapante Huapante (boy), Hacienda Santa Lucia with a temperature of $13^{\circ} \mathrm{C}$ and an altitude of 2793 meters above sea level ending in the canton Pelileo parish Salasaca presenting a temperature of $17^{\circ} \mathrm{C}$ and an altitude of 2600 meters above sea level. The equipment and materials that were used were sows Yorkshire (age 6 months and older, as well as physical materials was used as cane biometric, camera, tape, biometrics, SIUI $6.5 \mathrm{MHz}$ ultrasound, ultrasound gel, Marker and Pepel industrial for the study was carried out in sows of race Yorkshire in their different physiological states, from different places of origin. This work evaluated at the last rib to 4.6 and $8 \mathrm{~cm}$ of the line for both the right and the left, the thickness of the backfat using an echograph SIUI at a frequency of $6.5 \mathrm{MHz}$. In addition, a morphometric study was made of the animals that included height at the withers (AC), height at the croup (AEG), width of rump later (IIAS), body length (LEI), height back sternal (ADE), thoracic perimeter (PRT), perimeter of cana (PCA) and bicostal perimeter (PB), all these data became a normality test, followed by a correlation study that finally ended with a multiple regression analysis that led to the model of the equation with the reached the fulfilment of this research.

A study of the construction of predictive models not applies treatments; however, the study considered some grouping factors as age, physiological condition and to reduce noise in the model. In the present investigation was applied to the design of predictive correlations. To evaluate the response variable zoometricas different measures were taken within which is the height at the withers (AC), height at the croup (AEG), width of the croup (IIAS), body length (LEI), raised dorsoesternal (ADE), thoracic perimeter (PRT), perimeter of the sugar cane ( PCA), bicostal perimeter (PBC) of each animal in their different physiological states; then with the help of 6.5 $\mathrm{MHz}$ frequency SIUI Ultrasound, are valued at the last rib to 4, 6 and $8 \mathrm{~cm}$ from the middle line both the right and left the thickness of fat Dorsal. The variable to estimate was the backfat and through this to give a more objective measure of body condition. With the morpho metric measures zoometricos indices were calculated. The zoometricos measures and indices were subjected to a normality test the same that allowed to observe if the data obtained in the sample were normally distributed and consequently to be viable a parametric analysis and that were handled according to the number of data (Kolmogorov Smirnov $>50$ and Shapiro Wilk $<50$ ), then were correlated with the fat through the analysis of PEARSON through the procedure PROC CORR of SAS (SAS University Edition 2016). Logistic regression models were constructed through the stepwise procedure PROC REG of the SAS. In addition were evaluated in the model the inclusion of fixed and random variables. Fixed variables were considered as the age and physiological status and how random variables were considered the farm, through the MIXED procedure of SAS. The criteria AIC (The Akaike information criterion) And BIC (Bayesian information croterium) were considered to select the best model.

\section{Results}

\section{Test for Normality bristles of 6-I 2 months}

All the variables that corresponded to the group of animals between 6 to 12 months of age were subjected to the Shapiro-Wilk test for normality $(\mathrm{n}<50)$ to be able to identify the normal distribution of the data collected (Table 1). For the variable height at the withers (AC), height at the croup (AEG), width of rump (IIAS), body length (LEI), height back sternal (ADE), thoracic perimeter (PRT), bicoastal perimeter (PB) and live weight (LW) presented a value of $\mathrm{p}>0.05$, which shows that there is no statistical significance, for it accepts its normal distribution. On the other hand, variables such as age (E), perimeter of cane (PCA), back fat thickness right to 4,6 and $8 \mathrm{~cm}$ (GDD4, DRM6 and DRM8) of the middle line, back fat thickness left to 4, 6 and $8 \mathrm{~cm}$ (GDI4, GDI6 and GDI8) of the middle line and protein food (PC) showed values with statistical significance $(p<0.05)$, as a consequence, these variables did not show a normal distribution. This analysis allows us to identify the variables that can be subject to a parametric study based on their normal distribution; the measures of central tendency (mean and median) were similar for each variable. With regard to the variables and, AC, AEG, AIIS, LEI, ADE, PRT, PBC, PV and PC kurtosis values were lower than -0.5 indicates a distribution curve with platicurtico behavior with accumulation to the left with respect to the average according to the value of asymmetry. While variables such as PCA, GDD4, DRM6, DRM8, GDI4, GDI6 AND GDI8 presented Kurtosis greater than 0.5 related to a normal distribution curve leptocurtica, which, according to its asymmetry builds up to the right with respect to the media, while the standard deviation (a measure of dispersion) presented minimum values of 0.21 and maximum of 21.8 .

\section{Bristles of 13-18 months}

All the variables that corresponded to the group of animals between 12 to 18 months of age were subjected to a Kolmogorov-Smirnov test for normality $(n>50)$ to be able to identify the normal distribution of the data collected.

For the variables width of rump later (IIAS), body length (LEI), height dorzo sternal(ADE), thoracic perimeter (PRT) and live weight (LW) presented a value of $p>0.05$, which shows that there is no statistical significance, for it accepts its normal distribution. On the other hand, the variables age (E), height at the withers (AC), height at the croup (AEG), perimeter of cana (PCA), perimeter (PBC), bicostal thickness right in 4.6 and $8 \mathrm{~cm}$ (GDD4,GDD6 and DRM8) of the middle line, thickness left in 4.6 and $8 \mathrm{~cm}$ (GDI4,GDI6 and GDI8) of the middle line and protein food (PC) showed values with statistical significance $(\mathrm{p}<0.05)$, as a consequence, these variables did not show a normal distribution (Table 2).

This process leads to the identification of the variables that may be subject to a parametric study based on their normal distribution; the measures of central tendency (mean and median) were similar for each variable. With regard to the variables and, LEI, PRT and PV values of kurtosis were lower than -0.5 indicates a distribution curve with platicurtico behavior with accumulation to the left with respect to the average according to the value of asymmetry.

While variables such as iais, PCA, DRM6, DRM8, GDI4,GDI6 and GDI8 presented Kurtosis greater than 0.5 related to a normal distribution curve leptocurtica, which, according to its asymmetry builds up to the right with respect to the media. In addition to the variables $\mathrm{AC}, \mathrm{AEG}, \mathrm{ADE}, \mathrm{PBC}, \mathrm{DRM}$ and $\mathrm{PC} 4$ are found in a range 
$(-0.5$ to 0.5$)$ which gives it a mesocurtico behavior in relation to the average, with respect to the asymmetry describes an accumulation of data to the right side of the media with the exception of the variable PBC has distribution to the left, while the standard deviation (a measure of dispersion) presented minimum values of 0.30 and maximum of 15.86

\section{Older sows to 18 months}

All the variables that corresponded to the group of animals over 18 months of age were subjected to the Shapiro-Wilk test for normality $(\mathrm{n}<50)$ to be able to identify the normal distribution of the data collected (Table 3). For the variable height at the croup (AEG), body length (LEI) and sternal back height (ADE) presented a value of $p>0.05$, which shows that there is no statistical significance, for it accepts its normal distribution. On the other hand, the variables age (E), height at the withers (AC), width of rump later (IIAS), thoracic perimeter (PRT), perimeter of cana (PCA), perimeter (PBC), bicostal thickness right in 4.6 and $8 \mathrm{~cm}$ (GDD4,GDD6 and DRM8) of the middle line, thickness left in 4.6 and $8 \mathrm{~cm}$ (GDI4,GDI6 and GDI8) of the middle line, live weight (PC) and protein food (PC) showed values with statistical significance $(\mathrm{p}<0.05)$, as a consequence, these variables did not show a normal distribution. Through this study it was possible to identify the variables that can be subject to a parametric study based on their normal distribution, as can be seen in Table 4; the measures of central tendency (mean and median) were similar for each variable. With regard to the variable i read the values of kurtosis were lower than -0.5 indicates a distribution curve with platicurtico behavior with accumulation to the left with respect to the average according to the value of asymmetry.

While variables such as E, IAIS, PRT, PCA, PBC, PV, DRM4, DRM6, DRM8, GDI4, GDI6 and GDI8 presented Kurtosis greater than 0.5 related to a normal distribution curve leptocurtica, which, according to its asymmetry builds up to the right with respect to the media. With respect to the variables AC, AEG, ADE and PC have a mesocurtica curve behavior because their values are found in a range (-0.5 to 0.5$)$, in the variables $\mathrm{AC}$ and ADE you can observe an asymmetry with accumulation of data to the left, on the other hand the opposite happens with AEG and PC variables that have a build up toward the right with respect to the media.

\section{Pearson's correlation analysis}

Pearson's correlation analysis (Table 1) and (Table 2), showed high correlation values (R2>0.93) for the variables backfat thickness of right and left (GDD and GDI), when they were taken at 4, 6 and 8 $\mathrm{cm}$ of distance in relation to the dorsal line to the height of the last rib, and a highly significant probability $(\mathrm{p}=0.00)$; higher values to which appointment ${ }^{8}$ Who manifests values for $\mathrm{R} 2=0.55$. Which means that there is no variablidad in such data is, the value will be the same as the measure in 4.6 and $8 \mathrm{~cm}$ either both the right and left, always taking into account the animal's position and height above as well as mentioned. ${ }^{7}$ At the $14^{\text {th }}$ rib as to not be in the right place will not be able to perceive with the ultrasound machine the thickness of the back fat (Table 3).

\section{Bristles - 12 months}

In the correlation analysis for the variable thoracic perimeter was obtained a value of $\mathrm{R} 2$ of 0.92 , which reflects a high correlation with $\mathrm{PBC}$ and $\mathrm{PV}$ and a value of $\mathrm{p}=0.000$ indicating a highly significant probability in comparison to that which exposes Gutierrez ${ }^{12}$ who says that the values had a simple degree of significance. Well, these results can be exposed that the greater the weight of the animal, the greater his thoracic perimeter and bicostal on the contrary will do the opposite. On the other hand, the variable height at the withers (AC) showed a high correlation $(\mathrm{R} 2=0.86)$ with AEG, LEI, ADE, PRT, PCA, PBC and $\mathrm{PV}$ and a value of $\mathrm{p}=0.000$, indicating a highly significant probability between these variables. What we can say that the size and the proportionality of the sow are fully related for example to greater height at the withers (AC) higher live weight (LW). While for the variable height back sternal (ADE) reported a high correlation $(\mathrm{R} 2=0.88)$ with PRT, $\mathrm{PBC}$ and $\mathrm{PV}$ and a significance of $\mathrm{p}=0.000$; this manifests that the sternal back height variable is going to be directly proportional to the variables of PRT, PBC and PV. The variable bicostal perimeter $(\mathrm{PBC})$, only presented a high correlation $(\mathrm{R} 2=0.86)$ with live weight $(\mathrm{LW})$ with a highly significant probability $(\mathrm{p}=0.000)$. Given this result we can mention that the bicostal perimeter is directly proportional to the body weight well, fat sows in both variables were increased, and it is exactly the same in pregnant sows because as the gestation period progresses the PBC will increase and therefore the PV also.

For the variable height at the croup (AEG) found a high correlation $(\mathrm{R} 2=0.84)$ with $\mathrm{ADE}, \mathrm{PRT}$ and $\mathrm{PV}$ and a value of $\mathrm{p}=0.000$ indicating a highly significant probability, as mentioned ${ }^{13}$ To be a high correlation among these variables, although it is true while the proportion of the animal is greater, the greater your AEG, ADE, PRT and PV. The age variable showed a high correlation $(\mathrm{R} 2=0.77)$ with $\mathrm{AC}, \mathrm{ADE}, \mathrm{PRT}$ and $\mathrm{PV}$ and a highly significant probability $(\mathrm{p}=0.00)$. This means that older pigs presented higher values for your zoometricas measures related to their body proportion and the opposite will happen if there is less age. Finally, for the variable length Index (LEI) had a correlation of 0.77 with PRT and PV with a value of $p=0.00$. Which explains clearly that the proportion of the sow the same that is valued by the TRP and LEI will give rise to the PV of the sow?

\section{Bristles of I3-18 months}

The Pearson correlation study showed high correlation values (R2> 0.92)for backfat thickness variables left and right( GDD and GDI) taken at the level of 4,6 and $8 \mathrm{~cm}$ from the midline, in addition to a value of $\mathrm{p}=0.000$ indicating a highly significant probability that states that there is equity in the value measured either to the right or left side, however Chavarría ${ }^{8}$ Emits a criterion by highlighting that the left side brings with higher quality for the measurement of the thickness of the subcutaneous fat. While for the variable thoracic perimeter $(\mathrm{PRT})$ indicated a value of $(\mathrm{R} 2=1.00)$ with $\mathrm{PV}$ and a highly significant probability $\mathrm{p}=0.000$. These variables will be directly related which supports the concept of designating the live weight through the taking of measures of thoracic perimeter through the tape biometrics. On the other hand the variable height at the withers (AC) presented a high correlation $(\mathrm{R} 2=0.94)$ with $\mathrm{AEG}$ and a value of $\mathrm{p}=0.000$ indicating a highly significant probability. Which explains why the size of the pig is directly related to greater AC more AEG.

\section{Older sows to 18 months}

Through the analysis of Pearson could be determined for the variables of back fat thickness of both the right and left (GDD and GDI) at a height of 4,6 and $8 \mathrm{~cm}$ a value of $\mathrm{R} 2>0.89$ which gives a high correlation in conjunction with a value of $\mathrm{p}=0.000$ indicating a highly significant probability. However ${ }^{14}$ with regard to the measurement is made at the 10th thoracic vertebra, taking into account that the fat does not manifest itself in an equitable manner throughout your journey 
as mentioned. ${ }^{15}$ In addition to the variable height at the withers (AC) was presented a high correlation $(\mathrm{R} 2=0.92)$ with $\mathrm{AEG}$ and a value of $\mathrm{p}=0.000$ indicating a highly significant probability. Which explains why the size of the pig is directly related to greater AC more AEG, happening otherwise if it is less than the variable $\mathrm{AC}$. The variable thoracic perimeter (PRT) indicated a high correlation $(\mathrm{R} 2=0.90)$ with $\mathrm{PBC}$ and PV and a highly significant probability of $\mathrm{p}=0.000$. For the variable bicostal perimeter $(\mathrm{PBC})$ showed a value of $\mathrm{R} 2=$ 0.80 with $\mathrm{PV}$ and a value of $\mathrm{p}=0.000$ indicating a highly significant probability. Given these results, the PRT is relcionado directly with the PV and PBC because while increase the PRT will increase the PBC what happens with pregnant animals to greater PBC greater PV as manifested Quintero $\mathrm{J},{ }^{16}$ who evaluated sows to the 45,85 and 115 however he also mentions that the assessment of the thickness of fat is essential, as the PV can be given simply by fat and not muscle, however (Cool,D. 2014) suggests a diet ad libitum to improve the PV and back fat thickness (Table 4).

With the data obtained were able to identify the zoometricos indexes both of proportionality (height at the withers/ body length *100) and body (body length/thoracic perimeter*100) between the ages of 6-12, 13-18 and >18 months, the same as subject to a normality test and how relevant the coefficient of variation (CV) yielded the following results.

\section{Multiple regression analysis}

It allows us to estimate a possible relationship between a dependent variable and several independent. However, its importance lies in the identification of explanatory variables is to say helps to create a model where you select the variables that may influence the response, discarding those that do not provide information, ${ }^{17}$ So in Table 5 presents the following models.

\section{Discussion}

According to Hurtado ${ }^{13}$ the coefficients of variance of less than $12 \%$ show little variability found in these animals, as well in the following tables we can find values that fluctuate between $0.07-0.11 \%$ which is interpreted as a variability is much smaller than the one located by Hurtado. ${ }^{13}$ On the other hand, a comparative level with the article quoted by Hurtado $^{13}$ it was determined that the bristles evaluated presented, in the age of 6-12 months a percentage of $5.97 \%$, from 13 to 18 months a percentage of $7.06 \%$ and increased to 18 months a percentage of $6.08 \%$, minors in relation to the average reported by Hurtado $^{13} 12.26$ and $7.02 \%$ with respect to the coefficient of variance. With regard to these indices refers is the bristles evaluated have less proportionality in the regions of the body. Once exposed the different models, for the selection of the most appropriate took into account average values of R2, well this enables us to assess which is closer to the reality, taking into account the model that best fits the data as well as mentioned ${ }^{8}$ Who obtained values of $\mathrm{R} 2$ of 0.55 however the study opted for a $\mathrm{R} 2=0.2791$ that is not as close to unity as the of Chavarría. ${ }^{8}$ Which means that the model is the best among the candidate models, in the sense that provides the closest approximation to reality or to the true model. The model that best fits the data, you might change depending on the sample size, given that, with a larger sample size, the parameters of a model can be estimated more reliably as suggested. ${ }^{18}$
Another point that was taken into account were the variables that engage better results, in this way you can chose the model with three variables as it covers height to wing cross (BC), thoracic perimeter (PRT) and number of Births (deliveries) than are variables that are involved in a general manner with the zoometria de la cerda however to this choice exists ${ }^{3}$ That mentions that to choose a model to take into account the genetic group, year, sex and the interactions between these; well the three variables are correlated with all of the studied variables so that the height at the withers (AC) is related to the length of the extremities (PCA) and the height of the chest, reasons that also determine the sex of the animal and the factors for each race predominates height at the croup (AEG), width of rump later (IIAS), body length (LEI). The height at the withers also interacts with the development of the chest and the members here are spelled out the back sternal (ADE).

On the other hand the variable thoracic perimeter (PRT) encompasses a high correlation with bicostal perimeter (PBC) and live weight (LW) coinciding with Hurtado ${ }^{13}$ Who brings that possess a degree of significance $p=<0.05$, while the number of births would be measured as an indicator of age as bristles in a greater number of older will however not always the same. In addition, another reason why this model was selected was because it took into account the criteria the Akaike information criterion (AIC) and Bayesian information criterion (BIC) criteria that are within the statistical analysis, as well as do Martinez $\mathrm{R}^{3}$ Who uses the Generalized Linear Models procedure of the SAS statistical analysis package, another is also Cepero ${ }^{19}$ Who used the SPSS statistical package, which are statistical models that provide the correlation and multiple regression analysis according to a database, in this way highlights the criterion AIC that provides a penalty to the sobreparametrizacion, why not chose too many variables to the model, criterion AIC is not intended to select the correct model and try to obtain the model to provide better predictions between existing ones. It can be shown that, assuming that the true model can be approximated arbitrarily well with estimates by increasing the sample size, the AIC is efficient, choosing the model that provides (on average) better predictions. The BIC assumes that the real model is included in the set of candidate models, and measures the degree of certainty that a certain model is the true model generator of the data. (Díaz, 2011) $)^{18}$

$\mathrm{R} 2=0.2791$

Fat $=\mathrm{Y}+($ AC $*$ ACYANOPSIA $)+($ PRT $*$ NPARTOS PRTVD $)+(*$ NPARTOSVD)

Fat $=-0.013270 .35665+(-*$ ACYANOPSIA $)+(0.01808 *$ PRTVD $0.05336+(-)$ *NPARTOSVD)

However $^{3}$ for the equation of fat only took into account the fat and the current weight, but I believe that this is not convenient as it is to obtain the value of the back fat without its measurement ${ }^{20,21}$ used to change the value of final weight and body condition to estimate the amount of $\mathrm{kg}$ of meat to the channel which is not desirable because with this equation model what you want to do is to estimate the body condition. Once applied this model in the data studied was obtained a distribution as it displays the Table 5. The same that gives way to a classification for the prediction of body condition as indicated

\section{Conclusions}

Through the taking of encontaron zoometricas who is highly correlated with a value of $\mathrm{p}=0.000$ and the morphometric indices 
calculation it was possible to estimate the thickness of backfat in centimeters, which gave way to the classification of the body condition in which values were found ranging from 3-5. With regard to the correlation existing between the zoometricos found a value of R2 $>0.77$ giving a high correlation with values of $p=0.000$ highly significant; on the other hand, with regard to the fat found a high correlation of $\mathrm{R} 2>89$ and a value of $\mathrm{p}=0.000$ highly significant. Once evaluated the zoometricas measures by the different analysis it is concluded that the measures height at the withers, thoracic perimeter and the number of deliveries, conform to a prediction model based on a multiple regression analysis $(\mathrm{R} 2=0.2791)$ to estimate the value of the back fat thickness and estimate the body condition of sows Yorkshire from 6 months of age.

\section{Acknowledgements}

None.

\section{Conflict of interest}

Author declares there is no conflict of interest.

\section{References}

1. ASPE. Hog Statistics 2016. 2012.

2. AGROCALIDAD. National Survey Sanitary of Farms of Porcine Cattle-2010. Quito, Ecuador. 2011.

3. Martinez R, Salmerón F, Lopez M. Estimated heritability and comparison of genotypes cigars in pigs of the races Duroc, Landrace and Yorkshire and in reciprocal crosses of the races Landrace and Yorkshire, for backfat and weight to 154 days. Scielo. 2006;16(2)

4. Sanudo, C. Morphological assessment of domestic animals. Editorial SEZ: Spain; 2009.

5. Maple LE. Library practice of Animal Husbandry. Mexico DF: Tlalpan: 1987.

6. Charette R. Body condition evaluation in sows. ELSEVIER 1996;46(2):107-115.

7. Carrizosa D. Evaluation of the real-time ultrasound to predict the muscle and fat composition of the channel of the Iberian pig and the influence of the power in the characteristics of the fatty tissue. DIALNET: Spain; 2016.

8. Chavarría E. In vivo estimation of the channel swine by the method of ultrasonography in the period from October to November of the year 2014. RIUL. National Autonomous University of Nicaragua, Veterinary Medicine, Nicaragua. 2015.

9. Velásquez A. Measuring the thickness of backfat and loin eye area in channels of wild boar (Sus scrofa L) their relationship with the amount of fat and muscle. Universidad Austral de Chile; 2008.

10. Perez, Rodriguez V. Evaluation and Grading pig. Pig production. 2014;21.

11. Sanz M, Garcia A, Wennberg J. Assessment of the state of the sow. 2007.

12. Gutiérrez M. Variance components for growth traits and the thickness of the backfat in Cuba Duroc pigs. Pig production. 2011;18(2).

13. Hurtado CG. Morphological Study of the Creole pig in Apure state, Venezuela. pig Production. 2004;(03).

14. Mota D, Spilsbury M, Ramirez R, et al. Effect of the loss of fat and weight on the reproductive performance of lactating primiparous sows fed with three different types of diets. Scientific Journal, 2004;14(1).

15. Barceló J. Control of the body condition of sows based on the thickness of the backfat. Infcerd: 2005.

16. Quintero JE. Influence of the backfat thickness on reproductive parameters in hybrid lines and pure female pig on the farm the Sarita in the municipality of Medellin. De La Salle University Faculty of Agricultural Sciences program of animal husbandry. Bogotá DC. 2010.

17. Smith O. swine production system. IREGEP specialty livestock, Colegio de Postgraduados.Montecillo, 2009.

18. Díaz FF. Selection of models by using criteria of information in factor analysis. Theoretical and Computational aspects. University of Granada: Granada; 2011.

19. Cepero Y. Measures of fat thickness as a prediction of the content of meat of pigs. Gale Academic Onefile. 2004;14:3.

20. Mejia CM. Estimate in vivo performance of pig carcases by ultrasonography. Mexico: 1999.

21. UNNE. Animal production. (UNNE, Producer). Introduction to Animal Production. 2011. 